\title{
Characterization of $\mathrm{LiMn}_{2} \mathrm{O}_{4}$ cathodes by electrochemical strain microscopy
}

D. O. Alikin, A. V. levlev, S. Yu. Luchkin, A. P. Turygin, V. Ya. Shur, S. V. Kalinin, and A. L. Kholkin

Citation: Appl. Phys. Lett. 108, 113106 (2016); doi: 10.1063/1.4943944

View online: http://dx.doi.org/10.1063/1.4943944

View Table of Contents: http://aip.scitation.org/toc/apl/108/11

Published by the American Institute of Physics

\section{Articles you may be interested in}

Li transport in fresh and aged LiMn2O4 cathodes via electrochemical strain microscopy Appl. Phys. Lett. 118, 072016072016 (2015); 10.1063/1.4927816

Local probing of ionic diffusion by electrochemical strain microscopy: Spatial resolution and signal formation mechanisms

Appl. Phys. Lett. 108, 053712053712 (2010); 10.1063/1.3460637

Imaging space charge regions in Sm-doped ceria using electrochemical strain microscopy

Appl. Phys. Lett. 105, 201602201602 (2014); 10.1063/1.4901102

Electrochemical strain microscopy time spectroscopy: Model and experiment on LiMn2O4

Appl. Phys. Lett. 118, 055101055101 (2015); 10.1063/1.4927747

Delineating local electromigration for nanoscale probing of lithium ion intercalation and extraction by electrochemical strain microscopy

Appl. Phys. Lett. 101, 063901063901 (2012); 10.1063/1.4742933

Scanning thermo-ionic microscopy for probing local electrochemistry at the nanoscale

Appl. Phys. Lett. 119, 205110205110 (2016); 10.1063/1.4949473
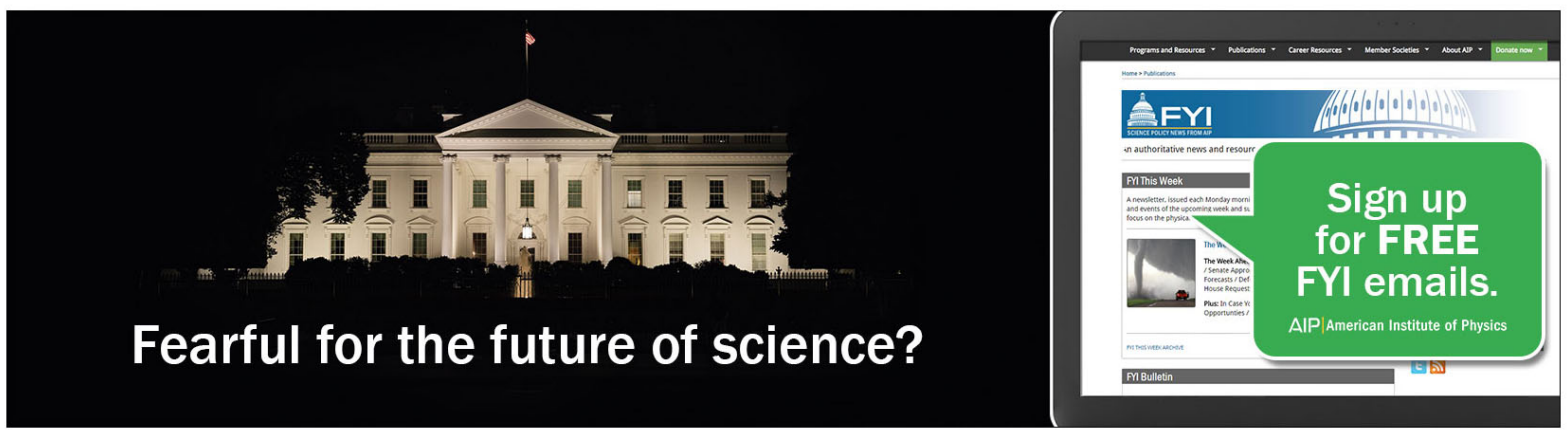


\title{
Characterization of $\mathrm{LiMn}_{2} \mathrm{O}_{4}$ cathodes by electrochemical strain microscopy
}

\author{
D. O. Alikin, ${ }^{1}$ A. V. levlev, ${ }^{2,3}$ S. Yu. Luchkin, ${ }^{4}$ A. P. Turygin, ${ }^{1}$ V. Ya. Shur, ${ }^{1}$ S. V. Kalinin, ${ }^{2,3}$ \\ and A. L. Kholkin ${ }^{1,4}$ \\ ${ }_{1}^{1}$ Institute of Natural Sciences, Ural Federal University, 51 Lenin Ave., Ekaterinburg 620000, Russia \\ ${ }^{2}$ The Center for Nanophase Materials Sciences, Oak Ridge National Laboratory, Oak Ridge, \\ Tennessee 37831, USA \\ ${ }^{3}$ Institute for Functional Imaging of Materials, Oak Ridge National Laboratory, Oak Ridge, \\ Tennessee 37831, USA \\ ${ }^{4}$ Physics Department and CICECO Aveiro Institute of Materials, University of Aveiro, \\ 3810 193, Aveiro, Portugal
}

(Received 16 November 2015; accepted 2 March 2016; published online 15 March 2016)

\begin{abstract}
Electrochemical strain microscopy (ESM) is a scanning probe microscopy (SPM) method in which the local electrodiffusion is probed via application of AC voltage to the SPM tip and registration of resulting electrochemical strain. Here, we implemented ESM to measure local strain in bulk $\mathrm{LiMn}_{2} \mathrm{O}_{4}$ cathodes of a commercial Li-battery in different states of charge to investigate distribution of Li-ion mobility and concentration. Ramped AC ESM imaging and voltage spectroscopy were used to find the most reliable regime of measurements allowing separating and diminishing different contributions to ESM. This is not a trivial task due to complex geometry of the sample and various obstacles resulting in less predictable contributions of different origins into ESM response: electrostatic tip surface interactions, charge injection, electrostriction, and flexoelectricity. Understanding and control of these contributions is an important step towards quantitative interpretation of ESM data. (C) 2016 AIP Publishing LLC. [http://dx.doi.org/10.1063/1.4943944]
\end{abstract}

Progress in secondary batteries and fuel cells is driven by advances in the development of more efficient materials for mobile ions storage and conduction. Crucial part of this process is thorough understanding of materials properties and microelectromechanical effects associated with the intercalation and deintercalation of mobile ions. Existing and emerging scanning probe microscopy (SPM) based techniques are able to probe a wide range of functional properties of ionically conducting materials at the nanoscale. ${ }^{1}$ Wider application and development of SPM-based techniques are important because of continuing reduction of the active particles' size, often towards the nanoscale. $^{2}$

Electrochemical strain microscopy $(\mathrm{ESM})^{3}$ is a SPM technique, which allows probing the local ionic mobility with resolution unattainable by traditional electrochemical methods. ${ }^{4}$ To date, the vast majority of ESM experiments were conducted on thin film samples with well defined geometry and limited number of possible contributions to the measured response. ${ }^{5}$ At the same time, ESM experiments on commercial Li-ion battery electrodes comprising the particles of ionic conductors embedded in a polymer binder matrix represent non-trivial problem due to complex geometry of the samples ${ }^{6}$ and consequent difficulties ${ }^{7}$ resulting in less predictable contributions of different origin into the strain response. ${ }^{8,9}$ From that point of view, we can generally talk about strain-based SPM (s-SPM) with a number of contributions, which must be separated in the experiments. ${ }^{10}$ Understanding and separation of these contributions is an important step from purely qualitative to quantitative data interpretation and analysis.

In this work, we measure and analyze ESM response on cross-sections of $\mathrm{Li}_{\mathrm{x}} \mathrm{Mn}_{2} \mathrm{O}_{4}$ cathodes with different $\mathrm{Li}$ concentrations and its distribution over the samples' surface.
Importantly, a complex signal dependence on distance from a current collector and state of charge of a battery was demonstrated. ESM excited with ramped AC voltage on a tip and ESM voltage spectroscopy results allowed us to show that the apparent ESM signal has a complex nature and real electrochemical contribution must be inspected carefully at low $\mathrm{AC}$ and DC voltages.

ESM measurements were performed on $\mathrm{LiMn}_{2} \mathrm{O}_{4}$ positive electrodes (cathodes) comprised $\mathrm{LiMn}_{2} \mathrm{O}_{4}$ ceramic particles embedded in poly(vinylidene fluoride) (PVDF) matrix with carbon black on the Al current collector (Figure 1). The electrodes were extracted from fresh cells in fully charged and discharged states, and mechanically separated from each other. A piece of the cathode was washed in dimethyl carbonate, embedded in an epoxy resin and polished. Note that even in fully charged and discharged states, remnant $\mathrm{Li}$ is always present in positive and negative electrodes, correspondingly. Lattice parameters of the cubic spinel $\mathrm{Li}_{\mathrm{x}} \mathrm{Mn}_{2} \mathrm{O}_{4}$

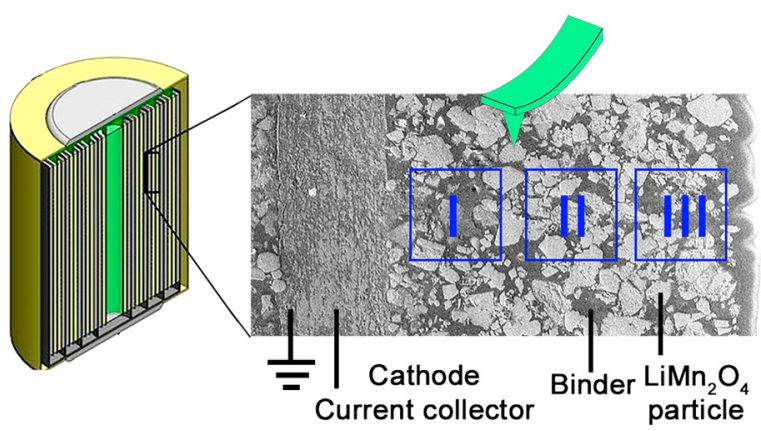

FIG. 1. Scanning electron microscopy image of the sample and scheme of pace in battery where fragment was cut and how ESM measurements were realized. Three regions between the Al current collector and the PVDF sepa rator are marked. 
samples were $0.8092 \mathrm{~nm}$ and $0.8178 \mathrm{~nm}$ in fully charged and fully discharged states, respectively. ${ }^{11}$

The sample's area was conventionally divided into three sections between the $\mathrm{Al}$ current collector and the PVDF separator (Figure 1) in order to study spatial inhomogeneity of ESM response (related to Li distribution) within the cathode. A number of scans from each section (up to 1030 particles in one section) were collected for the statistical variability.

ESM was implemented in the band excitation (BE) mode $^{12}$ using a commercial microscope (Cypher, Asylum Research) in combination with National Instruments PXI analog signal acquisition and generation electronics under ambient conditions. Samples were grounded through the metallic electrode $\mathrm{Al}$ current collector. Electrical contact between individual particles and $\mathrm{Al}$ current collector was provided by the carbon black network.

Pt coated cantilevers with about $2 \mathrm{~N} / \mathrm{m}$ spring constant and $70 \mathrm{kHz}$ fundamental resonance frequency were used as top moving electrodes. ESM imaging was made by applying $510 \mathrm{~V}$ AC voltage to the cantilever. Voltage spectroscopy ${ }^{13,14}$ with $30 \mathrm{~V}$ DC ramp and imaging with $30 \mathrm{~V}$ ramped $\mathrm{AC}$ at each point were additionally used.

ESM imaging and voltage spectroscopy: Figure 2 illustrates typical topography, contact resonance frequency, ESM response, and cross-section of the contact resonance frequency over the surface. The samples are composites comprising materials with significantly different mechanical properties, which give rise to resonance frequency shifts up to $30 \mathrm{kHz}$ between the $\mathrm{LiMn}_{2} \mathrm{O}_{4}$ particles and the binder (Figure 2(b)). Being measured at a single frequency at the contact resonance, this effect would cause a significant crosstalk and erroneous results. ${ }^{15}$ To avoid such errors, measurements were performed in BE mode, and the ESM response was extracted after fitting the resonance curve with the single harmonic oscillator model. ${ }^{12}$

Assuming that averaged over a single particle ESM signal reflects the average concentration of $\mathrm{Li}$ ions, we compared the magnitudes of ESM signal on the surface of different particles across the sample. Figures 2(d) and 2(e) represent histograms of the ESM response acquired on the samples in lithiated (Fig. 2(d)) and delithiated (Fig. 2(e)) states over three regions I, II, and III labeled in Figure 1. The statistics was obtained by averaging ESM signal over 3040 particles within one sample. One can see that on the lithiated sample, ESM response from regions (I) and (II) is relatively high, while response from the region (III) is notably lower. On the delithiated sample, relatively high response was observed only on the region (II), while both (I) and (III) regions showed very small and uniform response. The only difference between the samples is the concentration of mobile Li ions, so we believe that smaller response corresponds to lower Li concentration. Thus, the data in Figures 2(d) and 2(e) may represent spatial distribution of $\mathrm{Li}$ concentration in $\mathrm{Li}_{\mathrm{x}} \mathrm{Mn}_{2} \mathrm{O}_{4}$ particles between the $\mathrm{Al}$ current collector and the PVDF separator.

However, strain-based SPM can detect the AC electric field mediated strain of different origin: local molar volume variation due to ionic motion (Vegard contribution, or true ESM signal), piezoelectric and flexoelectric effects, and electrostriction. Resulting registered signal $(S)$ for the ionic materials can be expressed as follows: ${ }^{5,9,20}$

$$
S_{1 \omega}=S_{\text {Vegard }}+S_{\text {non Vegard }},
$$

where the first term represents Vegard electrochemical contribution and the second non-Vegard contribution.

For the $V_{a c} \cdot \sin (\omega t)$ excitation, these contributions can be expressed as ${ }^{19}$

$$
S_{V e g a r d}=\frac{e n D V_{a c} \sin (\omega t)}{f k_{B} T R_{0}},
$$

where $e$ is the elementary charge, $n$ is the concentration of mobile ions, $D$ is the diffusion coefficient of mobile ions, $f$ is the $\mathrm{AC}$ frequency, and $R_{0}$ is the radius of the tip-sample contact area. For non-Vegard contributions, the following expression applies

$$
\begin{aligned}
S_{\text {non Vegard }}= & {\left[\frac{2 l}{l^{*}} Q_{33} \chi \varepsilon_{0} P_{s}^{*}+\frac{2}{l^{*}} Q_{33} \chi^{2} \varepsilon_{0}^{2} V_{\text {surface }}\right.} \\
& \left.-\frac{1}{k} \frac{\partial C(z)}{\partial z} V_{\text {surface }}\right] V_{a c} \sin (\omega t),
\end{aligned}
$$

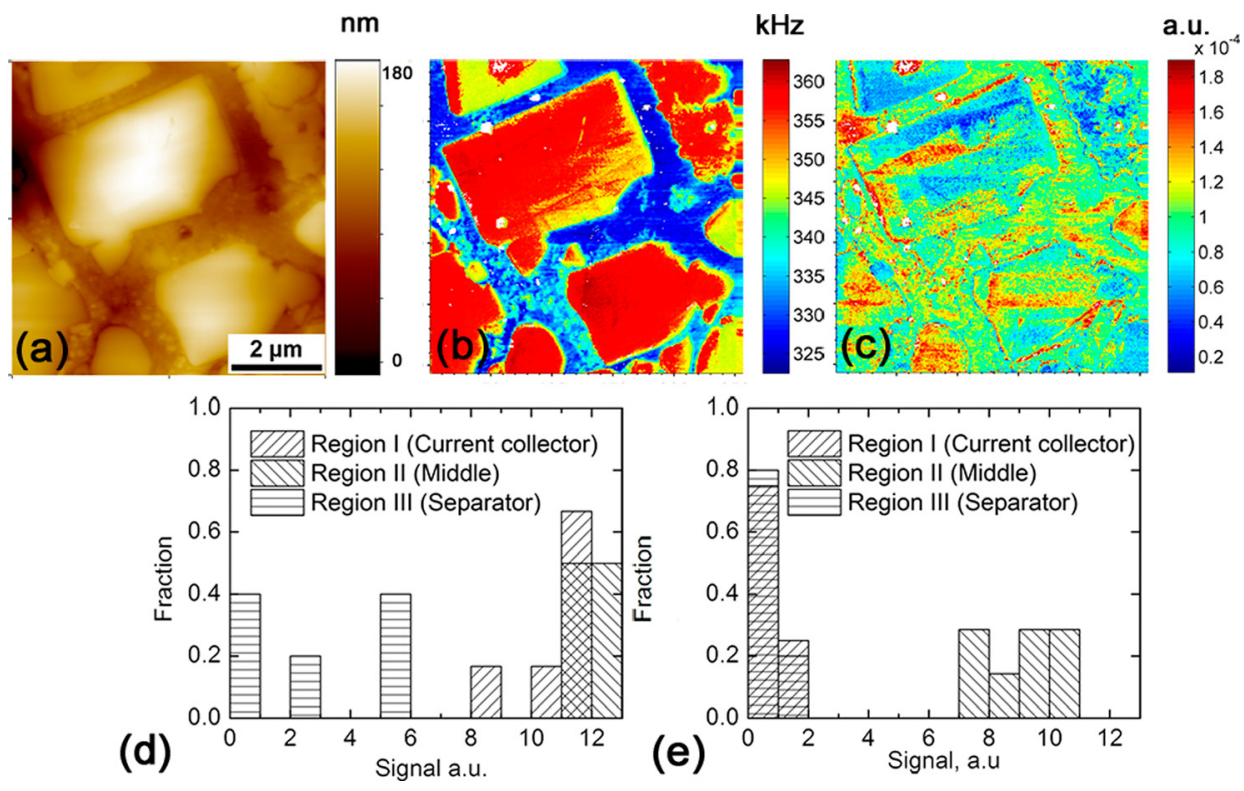

FIG. 2. Topography (a), BE contact resonance frequency map (b), ESM response (c) and cross section of the resonance frequency; normalized histo grams of the ESM signal distribution in lithiated (d) and delithiated (e) samples. 
where $l$ is the sample thickness, $P_{s}^{*}$ is the spontaneous polarization which exists permanently only in piezoelectrics but can appear due to flexoelectric effect and deformation potentials, $Q_{33}$ is the electrostriction coefficient, $\chi$ is the dielectric susceptibility, $l^{*}$ is the average penetration depth of $E_{a c}$ field, $V_{\text {surface }}$ is the surface potential, and $d C / d z$ is the capacitance gradient between the tip and the sample surface.

According to Eq. (2), Vegard contribution of ESM strain response depends on local concentration and mobility of $\mathrm{Li}$ ions in a material below the tip, which is determined by local diffusion coefficient. Li concentration can differ over the sample due to non-uniformity of charge/discharge process. Local diffusion coefficient may change at the grain boundaries and other microscopic defects. Additional non-Vegard contributions mentioned above $\mathrm{e}^{5,16}$ may complicate interpretation of the response. Thus, signal distribution in Figures 2(d) and 2(e) may be related not only to Li distribution.

To resolve the problem of non-Vegard contributions, voltage spectroscopy data are usually used as an additional method of characterization. ${ }^{14}$ In this method, a series of DC voltage pulses with increasing and decreasing heights are applied to the tip. Resulting ESM response is plotted as a function of the DC voltage applied on-pulse and represents hysteresis loop (Fig. 3(c)). The main parameter, which can be extracted from the hysteresis loop, is the area in biasstrain coordinates, which is proportional to local variation of Li concentration induced during the DC voltage cycle. The map of hysteresis loop area in Fig. 3(b) (so-called "reactivity map") looks smoother in comparison with ESM image (Fig. 3(d)) and does not show significant topography crosstalk. Signals demonstrate some similarity in distribution (Figs. 3(b) and 3(d), red marks).

In voltage spectroscopy, the ionic motion is caused not only by "fast" motion of ions induced by AC voltage but also by "slow" change of ion concentration under the tip induced by the DC voltage sweep. The area of ideal ESM hysteresis loop is believed to be a signature of the work of
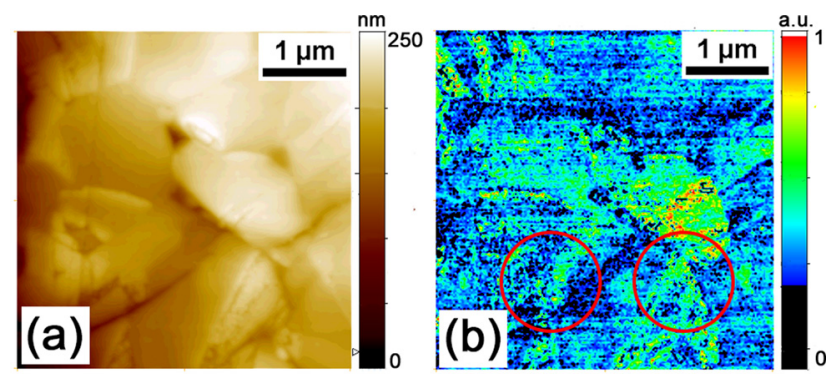

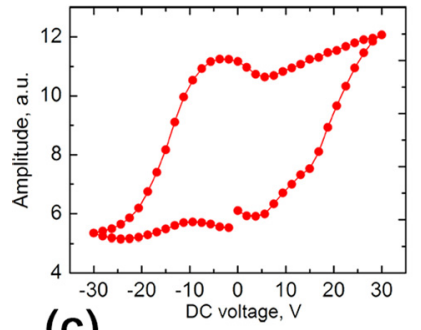

(c)

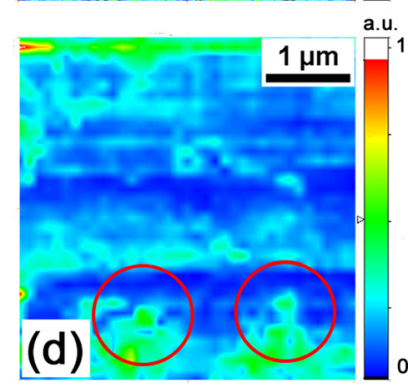

FIG. 3. Topography (a), ESM response (b), example of ESM hysteresis loop (c), and map of hysteresis loop area (d) obtained over the same area of the sample. Elements on the images marked by red circle are examples of simi lar behavior of ESM and voltage spectroscopy. local lithiation delithiation process under DC electric field. However, the loop can be closed not only when the applied DC does not change Li concentration due to lack of mobile ions but also when the strain relaxation time is smaller than the time between consequent pulses. Additional source for ambiguity is a possible local phase transition below the tip associated with the change of Li concentration during DC voltage sweep, ${ }^{9,21}$ which makes difficult to estimate local concentrations and diffusion coefficients from voltage spectroscopy data.

AC voltage amplitude dependences: Usually, lithiation delithiation of bulk cathode materials is performed at a low operation bias. ${ }^{17}$ In ESM, applied AC voltage directly determines the value of a registered signal, and an increase of AC voltage can significantly improve the quality of the obtained data. On the other hand, high voltage may result in the appearance of additional contributions. ${ }^{5,9,18}$ That is why we used ESM mapping with ramped AC voltage applied at each point to reveal how high AC voltage can influence the resulting signal. Figure 4 shows the spatial maps and extracted ESM amplitudes for both samples. ESM signal inside the particles demonstrates pronounced nonlinearity versus AC voltage, while theoretically, it is expected to be linear. ${ }^{19}$ In the lithiated sample, non-linearity appears between 3 and $5 \mathrm{~V}$ (Fig. 4(c)), while in the delithiated sample, it is more pronounced and appears between 15 and $20 \mathrm{~V}$ (Fig. 4(d)). The slope of the dependence changes in the delithiated sample, as shown in Figure 4(d) blue curve. In comparison, ESM response in PVDF matrix has a linear dependence on applied AC voltage, which indicates ferroelectric betta-phase of PVDF or electrically induced ferroelectric-like state.

One can clearly see from Eqs. (1) (3) that all contributions are linear with respect to $V_{a c}$. The possible explanations of obtained non-linearity is that the phase transition from the cubic $\mathrm{Fd} 3 \mathrm{~m} \mathrm{LiMn}_{2} \mathrm{O}_{4}$ to the tetragonal I41/amd $\mathrm{Li}_{2} \mathrm{Mn}_{2} \mathrm{O}_{4}$ taking place in $\mathrm{Li}_{\mathrm{x}} \mathrm{Mn}_{2} \mathrm{O}_{4}$ at $\mathrm{x}$ exceeding 1 (or, strictly
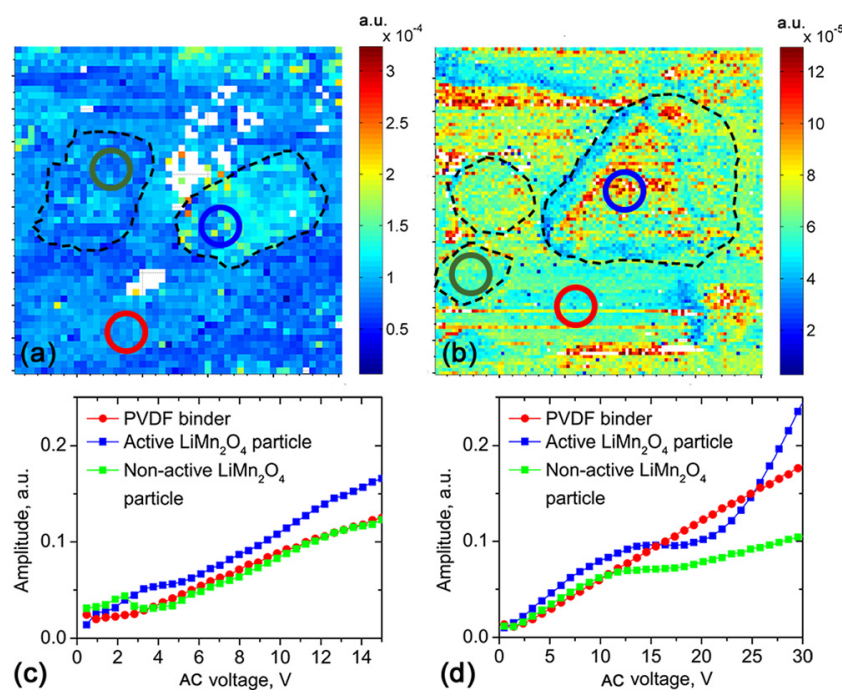

FIG. 4. ESM images (at $10 \mathrm{~V}$ AC) and dependence of the ESM signal on applied AC voltage in the lithiated (a) and (c) and delithiated (b) and (d) samples. The dashed black line on (a) and (b) indicates border of individual particles. Colors of circles on (a) and (b) correspond to the colors of AC dependencies on (c) and (d). Note that ESM signal on the lithiated sample is higher (factor $10^{4}$ vs $10^{5}$ on the delithiated sample). 
speaking, average $\mathrm{Mn}^{\mathrm{n}+}$ oxidation state reduces below 3.5). ${ }^{22}$ Being driven by the electric overpotential, it can locally occur under the tip during application of high AC voltage. Higher initial Li concentration must require smaller external field leading to overlithiation and the phase transition.

If this hypothesis stands, the response before the bending of the experimental curve corresponding to the cubic phase must have sharper slope than the one in tetragonal phase, according to Vegard coefficient. To solve this contradiction, change of electrostriction coefficient or surface potential $\left(V_{s p}\right)$ during phase transition can be assumed, but it will be rather speculative and requires additional experimental observations.

The observed behavior has one important consequence. Distribution of ESM signal in particles changes significantly when the applied voltage achieves non-linear region on experimental curve. ${ }^{21}$ Thus, results measured only at a fixed AC voltage may have different interpretation depending on the voltage. To extract spatial distribution of characteristic response-excitation curves from spectroscopic data, we used principle component analysis (PCA) and k-means clustering. ${ }^{23-25}$ Similar approach was recently used for statistical analysis of the micro-Raman data. ${ }^{26,27}$ PCA allows automated deconvolution of the vectors set $A_{i}$ (ESM response vs AC voltage curves) on the linear combination of orthogonal uncorrelated eigenvectors $v_{k}$ with weight/ loading coefficients $l_{i k}$

$$
A_{i}=l_{i k} v_{k} .
$$

Then eigenvectors are sorted in a descending order by variance. The first eigenvector contains the most statistically important information about system behavior, second the most important information after subtraction of the first one, and so on. In this manner, important patterns of system behavior are characterized by a few first eigenvectors and set
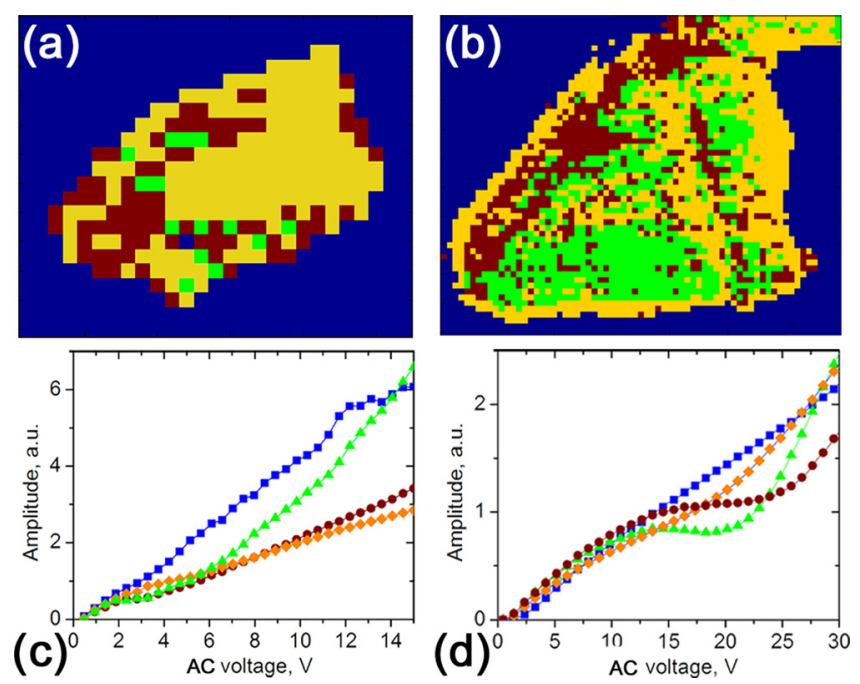

FIG. 5. Results of the automated $\mathrm{k}$ means clustering performed for the ESM images with linearly increasing AC voltage. Map of regions with dif ferent ESM signal behavior in the lithiated (a) and delithiated (b) sample, and correspondent average AC dependences (c) and (d). Colors on (a) and (b) correspond to colors on (c) and (d). of the corresponding loadings, ${ }^{21}$ while last eigenvectors are defined by noise and distortions only. Application of the $\mathrm{k}$-means clustering in multidimensional space formed by PCA weight coefficients allowed us to plot a map of the regions with fundamentally different spectra (Fig. 5). Note that PCA is performed on the three-dimensional dataset of ESM response as a function of amplitude of the applied AC voltage and spatial position. It clearly shows that non-linearity in ESM signal is localized only in several regions of the particle. These facts must be taken into consideration when comparing the results. Otherwise, different mechanisms of underlying response can be wrongly interpreted as equal.

In conclusion, in this work, we implemented ramped AC voltage ESM imaging and voltage spectroscopy to study local Li activity and its variation as a function of Li concentration. Non-uniform distribution of the response signal on $\mathrm{LiMn}_{2} \mathrm{O}_{4}$ particles was shown and attributed to different electrochemical activity of lithium in different regions of a particle. At the same time, it was experimentally shown that s-SPM response cannot be simply interpreted as proportional to mobility of Li ions due to multiple non-Vegard contributions including electrostatic forces, charge injection, and electrostriction. Distribution of ESM signal was found to change significantly with increase of AC amplitude, which was attributed to the phase transition from cubic $\mathrm{Fd} 3 \mathrm{~m}$ $\mathrm{LiMn}_{2} \mathrm{O}_{4}$ to tetragonal I41/amd $\mathrm{Li}_{2} \mathrm{Mn}_{2} \mathrm{O}_{4}$ phases accompanied by the Jahn Teller distortion. Proposed analysis of ESM signal distribution by principal components analysis allows distinguishing regions in particles with different signal behavior.

These results demonstrate that the voltage during ESM measurements must be carefully controlled to avoid undesirable contributions of the electromechanical non-Vegard coupling. Further theoretical development and experiments are required for the qualitative analysis of the observed phenomena.

The equipment of the Ural Center for Shared Use "Modern nanotechnology" UrFU was used. The research was made possible in part by UrFU development program with the financial support of young scientists. The work was partially supported by Government of the Russian Federation (Act 211, Agreement 02.A03.21.0006). V.Y.S. acknowledges financial support within the State Task from the Ministry of Education and Science of Russian Federation (Project No. 1366.2014/236).

A portion of this research (A.V.I. and S.V.K.) was conducted at the Center for Nanophase Materials Sciences, which is a DOE Office of Science User Facility. V.Y.S., D.O.A., A.I.L., and A.P.T. acknowledge CNMS user proposal (Project 2013-130).

The work was supported by the European Commission within FP7 Marie Curie Initial Training Network "Nanomotion" (Grant Agreement No. 290158). The authors gratefully acknowledge H.-Y. Amanieu and D. Rosato (Robert Bosch $\mathrm{GmbH}$ ) for providing the samples and useful discussion.

This work was developed in the scope of the project CICECO-Aveiro Institute of Materials (Ref. FCT UID/CTM/ 50011/2013), financed by national funds through the FCT/ 
MEC and, when applicable, co-financed by FEDER under the PT2020 Partnership Agreement.

${ }^{1}$ S. V. Kalinin and N. Balke, "Local electrochemical functionality in energy storage materials and devices by scanning probe microscopies: Status and perspectives," Adv. Mater. 22, E193 E209 (2010).

${ }^{2}$ B. Dunn, P. Liu, and S. Meng, "Nanoscience and nanotechnology in next generation lithium batteries," Nanotechnology 24, 420201 (2013).

${ }^{3}$ A. N. Morozovska, E. A. Eliseev, N. Balke, and S. V. Kalinin, "Local probing of ionic diffusion by electrochemical strain microscopy: Spatial resolution and signal formation mechanisms," J. Appl. Phys. 108, 053712 (2010).

${ }^{4}$ A. N. Morozovska, E. A. Eliseev, and S. V. Kalinin, "Electromechanical probing of ionic currents in energy storage materials," Appl. Phys. Lett. 96, 222906 (2010).

${ }^{5}$ S. V. Kalinin and A. N. Morozovska, "Electrochemical strain micros copy of local electrochemical processes in solids: Mechanism of imag ing and spectroscopy in the diffusion limit," J. Electroceram. 32, 5159 (2014).

${ }^{6}$ J. R. Wilson, J. S. Cronin, S. A. Barnett, and S. J. Harris, "Measurement of three dimensional microstructure in a $\mathrm{LiCoO}_{2}$ positive electrode," J. Power Sources 196, 34433447 (2011).

${ }^{7}$ H. Y. Amanieu, D. Rosato, M. Sebastiani, F. Massimi, and D. C. Lupascu, "Mechanical property measurements of heterogeneous materials by selec tive nanoindentation: Application to $\mathrm{LiMn}_{2} \mathrm{O}_{4}$ cathode," Mater. Sci. Eng. A 593, 92102 (2014).

${ }^{8}$ S. Y. Luchkin, K. Romanyuk, M. Ivanov, and A. L. Kholkin, "Li transport in fresh and aged $\mathrm{LiMn}_{2} \mathrm{O}_{4}$ cathodes via electrochemical strain micro scopy,” J. Appl. Phys. 118, 072016 (2015).

${ }^{9}$ H. Y. Amanieu, H. N. M. Thai, S. Yu. Luchkin, D. Rosato, D. C. Lupascu, M. A. Keip, J. Schroder, and A. L. Kholkin, "Electrochemical strain mi croscopy time spectroscopy: Model and experiment on $\mathrm{LiMn}_{2} \mathrm{O}_{4}$," J. Appl. Phys. 118, 055101 (2015).

${ }^{10}$ J. Li, J. F. Li, Q. Yu, Q. N. Chen, and S. Xie, "Strain based scanning probe microscopies for functional materials, biological structures, and electro chemical systems," J. Mater. 1, 321 (2015).

${ }^{11}$ H. Y. Amanieu, M. Aramfard, D. Rosato, L. Batista, U. Rabe, and D. C. Lupascu, "Mechanical properties of commercial $\mathrm{LixMn}_{2} \mathrm{O}_{4}$ cathode under different states of charge," Acta Mater. 89, 153162 (2015).

${ }^{12}$ S. Jesse, S. V. Kalinin, R. Proksch, A. P. Baddorf, and B. J. Rodriguez, "The band excitation method in scanning probe microscopy for rapid map ping of energy dissipation on the nanoscale," Nanotechnology 18, 435503 (2007).
${ }^{13}$ A. Kumar, F. Ciucci, A. N. Morozovska, S. V. Kalinin, and S. Jesse, "Measuring oxygen reduction/evolution reactions on the nanoscale," Nat. Chem. 3, 707713 (2011).

${ }^{14}$ Y. Kim, A. N. Morozovska, A. Kumar, S. Jesse, E. A. Eliseev, F. Alibart, D. Strukov, and S. V. Kalinin, "Ionically mediated electromechanical hys teresis in transition metal oxides," ACS Nano 6, 70267033 (2012).

${ }^{15}$ S. Jesse and S. V. Kalinin, "Band excitation in scanning probe micros copy: Sines of change,” J. Phys. D: Appl. Phys. 44, 464006 (2011).

${ }^{16} \mathrm{E}$. A. Eliseev, A. N. Morozovska, A. V. Ievlev, N. Balke, P. Maksymovych, A. Tselev, and S. V. Kalinin, "Electrostrictive and electro static responses in contact mode voltage modulated scanning probe micro scopies," Appl. Phys. Lett. 104, 232901 (2014).

${ }^{17}$ J. B. Goodenough and K. S. Park, "The Li ion rechargeable battery: A perspective," J. Am. Chem. Soc. 135, 11671176 (2013).

${ }^{18}$ J. S. Sekhon, L. Aggarwal, and G. Sheet, "Voltage induced local hysteretic phase switching in silicon,” Appl. Phys. Lett. 104, 162908 (2014).

${ }^{19}$ A. Tselev, A. N. Morozovska, A. Udod, E. A. Eliseev, and S. V. Kalinin, "Self consistent modeling of electrochemical strain microscopy of solid electrolytes," Nanotechnology 25, 445701 (2014).

${ }^{20}$ G. da Cunha Rodrigues, P. Zelenovskiy, K. Romanyuk, S. Luchkin, Y. Kopelevich, and A. Kholkin, "Strong piezoelectricity in single layer gra phene deposited on $\mathrm{SiO}_{2}$ grating substrates," Nat. Commun. 6, 7572 (2015).

${ }^{21}$ See supplementary material at http://dx.doi.org/10.1063/1.4943944 for Figure 1 which shows topography evolution under the DC voltage pulses. Figure 2 shows application of contour analysis to the ESM image of dis tinct particle. Figure 3 shows eigen vectors and main components obtained by PCA analysis.

${ }^{22}$ H. Berg, K. Goransson, B. Nolang, and J. O. Thomas, "Electronic structure and stability of the $\mathrm{LixMn}_{2} \mathrm{O}_{4}(0<\mathrm{x}<2)$ system," J. Mater. Chem. 9, 28132820 (1999).

${ }^{23}$ G. A. F. Seber, Multivariate Observations (John Wiley \& Sons, 2004).

${ }^{24} \mathrm{~N}$. Bonnet, "Multivariate statistical methods for the analysis of microscope image series: Applications in materials science," J. Microsc. 190, 218 (1998).

${ }^{25}$ S. Jesse and S. V. Kalinin, "Principal component and spatial correlation analysis of spectroscopic imaging data in scanning probe microscopy," Nanotechnology 20, 085714 (2009).

${ }^{26}$ A. V. Ievlev, M. A. Susner, M. A. McGuire, P. Maksymovych, and S. V. Kalinin, ACS Nano 9, 1244212450 (2015).

${ }^{27}$ V. Iberi, A. V. Ievlev, I. Vlassiouk, S. Jesse, S. V. Kalinin, D. C. Joy, A. J. Rondinone, and O. S. Ovchinnikova, "Graphene engineering by neon ion beams," Nanotechnology 27, 125302 (2016). 